\title{
MLS - EDUCATIONAL RESEARCH
}

http://mlsjournals.com/Educational-Research-Journal

ISSN: $2603-5820$

\section{Como citar este artículo:}

Mundet, L. B. \& Peña Muñoz, J. M. (2021). Percepción de competencias en las prácticas profesionalizantes e inserción laboral del Técnico en Redacción de Textos. MLS Educational Research, 5(2), 59-72. doi: 10.29314/mlser.v5i2.531.

\section{PERCEPCIÓN DE COMPETENCIAS EN LAS PRÁCTICAS PROFESIONALIZANTES E INSERCIÓN LABORAL DEL TÉCNICO EN REDACCIÓN DE TEXTOS}

\author{
Lina Beatriz Mundet \\ Universidad Internacional Iberoamericana (México) \\ mundet.lina@gmail.com·https://orcid.org/0000-0003-2561-9312 \\ Jesús Manuel Peña Muñoz \\ Universidad Internacional Iberoamericana (México) \\ jemape9@gmail.com·http://orcid.org/0000-0003-3568-9787
}

\begin{abstract}
Resumen. La investigación analiza la percepción que poseen los redactores de textos, egresados del Instituto Superior de Letras Eduardo Mallea, ISLEM, Argentina, en relación con las competencias adquiridas en las prácticas profesionalizantes y los requerimientos del acceso al empleo. El trabajo se inscribe en la Línea de Investigación Competencia Laboral, Educación y Empleo de la Universidad Internacional Iberoamericana, UNINI, México, y se basa en los aportes del enfoque de la socioformación y de la metodología sistémica de una organización. La hipótesis enfrenta las dos variables: a) percepción de los redactores egresados respecto de sus competencias adquiridas en las prácticas profesionalizantes y b) requerimientos de los empleadores, a fin de sopesar sus correspondencias e identidades. La metodología se enfoca en un estudio de campo, no experimental y transversal, correlacional, de corte cuantitativo, donde se buscan relaciones para verificar la hipótesis planteada. Se aplica un cuestionario que recoge la información de las dos variables citadas. La población es constituida por la totalidad de egresados de las promociones de la tecnicatura del ISLEM, representada por 51 (cincuenta y un) técnicos en expresión escrita con experiencia en el área. No hay selección de muestra, se realiza tipo censo poblacional. En los resultados, se obtienen coincidencias entre los dos tipos de percepciones con pocas variantes. Para finalizar se realiza la propuesta, donde se plantea un programa de formación continua de competencias ajustadas a los cambios detectados en el trabajo e indispensables para la inserción laboral del egresado redactor de textos del ISLEM.
\end{abstract}

Palabras clave: Competencias comunicativas, expresión escrita, alumno en prácticas, acceso al empleo.

\section{PERCEPTION OF COMPETENCES IN PROFESSIONALIZING PRACTICES AND LABOR INSERTION OF THE TECHNICIANS IN TEXT WRITING}

Abstract. The aim of the research is to analyse the perception of text writers graduated from the Instituto Superior de Letras Eduardo Mallea (ISLEM), in relation to professionalization practice competencies and whether or not they are appropriate for job placement requirements. The work is linked to the dynamics of 
application of the Line of Research Labor Competence, Education and Employment of the Universidad Internacional Iberoamericana and is based on the contributions of the socioforming and systemic methodology of an organization approach. The hypothesis relates two variables: a) the perception that the text writers have about the mastery reached at the time of the professionalization practices is related to the level of requirements experienced during the work exercise of the writing editor's skills and b) employers' requirements, in order to weigh their matches and identities. The methodology is focused on a field study, non-experimental and crosssectional, correlational, quantitative cut where relationships are sought in order to verify the hypothesis. A questionnaire is applied to collect information about both of the variables. The population is constituted by the totality of graduates from the ISLEM, represented by 51 technicians in text writing graduated with experience in the area. There is no sample selection, population census type is performed. In the results, coincidences between the two types of perceptions of graduates are obtained. To conclude, a proposal is made, which raises the layout of a continuing training program about competences subject to changes in the labor market, essential for the labour integration of all professional writers graduated from ISLEM.

Keywords: Communicative competencies, writing, professionalizing practices, labor insertion.

\section{Introducción}

La educación terciaria en la Argentina y en América latina en general, a diferencia de la universitaria, la cual suele orientarse hacia la investigación, asume el compromiso de formar técnicos profesionales que tengan el cometido de incorporarse laboralmente, en forma casi inmediata, en los lugares clave donde requieran de las competencias para las que fueron preparados. De ahí la necesidad de una constante revisión de los procesos, las funciones profesionales y los programas de formación para ir incorporando los cambios producidos a nivel interno y externo de sus contextos.

Una de las formas más plausibles de generar planes de estudio para una carrera, afirma González (2016), consiste en realizar un Análisis Situacional del Trabajo (AST); esto significa formar un taller multidisciplinar con académicos, empresarios, supervisores y expertos, cuyo objetivo se encamine a recabar información confiable acerca de las competencias que debe poseer el egresado para desempeñarse exitosamente en el campo laboral. Y si bien para el centro educativo los usuarios son la prioridad del accionar de sus integrantes, necesita para garantizar un eficaz servicio ofrecer las mejores carreras y, por ende, los mejores programas de estudio; de allí que cada cuatro años se evalúe la pertinencia de los programas educativos para adecuarlos mejor al acelerado ritmo tecnológico de estos tiempos.

Los organismos implicados en la formación de técnicos profesionales para la inserción laboral (centro educativo-Estado-empresas) están comenzando a comprender la importancia de aplicar algunas estrategias de cooperación: redes de conocimientos, equipos de trabajo, establecimiento de lineamientos concertados para el sector, entre otras. Así, los entes gubernamentales se avienen a integrar proyectos conjuntos a partir de pautas establecidas, producto de mesas de encuentro con los involucrados, apoyo estatal en metodologías, financiamiento de proyectos y acuerdos mutuos. Las investigaciones citadas en este artículo destacan que la vinculación entre los integrantes de esta tríada ayuda a enfrentar los cambios permanentes.

Si bien es cierto que se han conseguido avances importantes, los institutos de educación técnica presentan actualmente varias limitaciones en cuanto a capacitación del personal directivo y docente, infraestructura, aplicación del modelo educativo tradicional y problemas operativos concernientes a las prácticas profesionalizantes ( $\mathrm{PP}$ en adelante) vinculadas con el sector laboral. De acuerdo con el Instituto Nacional de Educación 
Tecnológica (2019), se advierten carencias de habilidades prácticas de los estudiantes/egresados a la hora de insertarse en el ámbito laboral, donde se incluyen tanto actividades prácticas dentro de los centros educativos, como experiencias de trabajo en establecimientos productivos. Respecto de la pertinencia de los contenidos, también aparecen brechas de formación en conocimientos específicos y una necesidad de actualización frente al requerimiento de la industria.

Para mantener la vigencia de los contenidos formativos, es imprescindible que las instituciones de formación profesional estén en contacto permanente con la realidad social y productiva, sus innovaciones y sus caducidades. Florez, Luraschi y Butti (2017) consideran clave que los estudiantes realicen una práctica donde experimenten situaciones lo más parecidas posible a lo real, a fin de integrar los aprendizajes en miras de consustanciarse con procesos de trabajo propios de la profesión. Bajo estas exigencias, las funciones de los directivos, docentes y estudiantes tendrán que centrarse en las PP, espacios curriculares transversales que hegemonizan el desarrollo de competencias técnicas adaptadas a las exigencias del mundo laboral.

Estas PP, consideradas como las más preciadas innovaciones educativas de los institutos técnicos, permiten al futuro profesional la construcción de conocimiento, ya que un egresado deberá enfrentarse a los problemas y exigencias surgidos a cada momento en el contexto donde se desempeñe. De esto se desprende que las instituciones de educación superior deben estar comprometidas en brindar estrategias para los cursantes, a fin de que estos internalicen saberes y habilidades, y a la vez experimenten en ambientes reales o simulados, reflexionen sobre la práctica y logren una comprensión significativa, amén de facilitar los medios y equipos para el acceso a la información más pertinente.

La investigación de Di Meglio, Barge-Gil, Camiña y Moreno (2019) señala que, para un desempeño efectivo de las habilidades, los estudiantes deberían asumir un rol activo con relación a sus aprendizajes, a partir de espacios de reflexión donde se enfatice la importancia del grupo y la mirada de los otros. Aplicar estrategias de resolución de conflicto en las tareas propias del mundo del trabajo posibilita a los estudiantes detectar los obstáculos, analizarlos, comprender sus causas y efectos. Esta actitud crítico-reflexiva ayuda a confrontar los conocimientos disciplinares con la realidad y a la vez profundizar sobre el porqué y el para qué de acciones y estrategias. Así, reconocer el conocimiento cuestionable y discutible permite a los cursantes repensarlo en vez de reproducirlo. Por ende, el conocimiento debe integrar la teoría-práctica mediante el análisis y la reflexión en la resolución de problemas, aprendiendo en el hacer y reflexionando sobre lo que hace.

Sobre este mismo tema, Fascendini, Guaita, Peralta y De Gennaro (2019) expresan la necesidad de articular los talleres de las PP a la estructura curricular de la formación, a los que catalogan como oportunidades válidas de la formación profesional por propiciar un aprendizaje compartido entre pares y abierto a la realidad del mundo del trabajo. Sin embargo, Silva et al. (2019) comprobaron que el desarrollo exclusivo de las competencias específicas y el descuido en el afianzamiento de las genéricas suele producir en los egresados dificultades de adaptación en la etapa inicial de su desarrollo profesional.

Estos requerimientos implican la necesidad de revisar las competencias de los estudiantes de la Tecnicatura Superior en la Redacción de Textos, del Instituto Superior de Letras Eduardo Mallea (ISLEM). En ese sentido, este artículo se propone presentar el análisis del contexto empírico y los resultados obtenidos del instrumento aplicado a la investigación que confronta dos variables: a) la percepción que los egresados del ISLEM tienen de las competencias adquiridas durante su formación como redactores -en concreto, en las $\mathrm{PP}-\mathrm{y} \mathrm{b}$ ) la percepción de las competencias exigidas en la inserción laboral. 


\section{Revisión literaria}

El devenir constante que vive la economía y el mercado mundial, sumado al alto grado de competitividad nacional e internacional, ha requerido de las organizaciones públicas y privadas implementar estrategias innovadoras y ajustadas a la realidad para sobrevivir; en consecuencia, las exigencias obligan a los empleadores a buscar talento humano con perfiles calificados con el fin de adaptarse a los cambios, esto es, individuos con dominio de competencias cada vez más especializadas que aporten valor al proceso de producción. Por ende, las organizaciones deben estar preparadas para lo que Pedreño (2017) denomina "el impacto disruptivo", esencial en la ordenación de los diversos sectores comerciales e industriales de cualquier sociedad. Ya es una evidencia palpable la industria 3.0 o 4.0, la big data, machine learning, entre otros, los cuales producen gran impacto en los diversos sistemas productivos.

Paralelamente, la mayoría de las acciones nacionales e internacionales reconoce la necesidad de potenciar la pertinencia del perfil profesional con las alternativas viables para la inserción que permita la consolidación del mercado laboral con la formación recibida en instituciones del sistema educativo. Sin duda, no es tarea fácil calibrar el trabajo de las empresas a las exigencias del contexto, pero ese esfuerzo no puede darse solo, porque requiere, como se dijo, de la alianza entre los entes que hacen vida en el sistema productivo, como los organismos del Estado, los centros de formación profesional y las asociaciones empresariales.

De ahí que no se deba responsabilizar solamente a las instituciones educativas, advierten Tomassiello y Zani (2016), de que las estructuras curriculares no se adapten a las gestiones profesionales o a los problemas globales de mercado. La esperanza de adaptación a lo nuevo está puesta en las prácticas interdisciplinares de grupos que puedan contribuir a estimular el surgimiento de las distintas visiones del problema que redunde en la actualización de los conocimientos con resultados positivos para los egresados y la institución.

Se parte de la idea de que las exigencias de la inserción laboral del técnico en redacción de textos devienen cada vez más complejas, ante el mayor desafío de las organizaciones y el talento humano. Por delante el egresado tiene el avance tecnológico, la brecha entre empresas grandes y pequeñas, competitividad, investigación, innovación, formación permanente, entre otros. Conocer la complejidad descrita y realizar la evaluación periódica de las competencias, por parte de los actores involucrados en la inserción de los nuevos profesionales del sector, permite ir estructurando un proceso de construcción de estrategias en cada organización y entre los implicados para evidenciar las dificultades y posibles acciones individuales y colectivas al emprender y verificar el éxito o fracaso de un programa.

La importancia de la evaluación de las competencias necesarias de los profesionales en redacción de escritos contribuye a mejorar la enunciación y puesta en marcha del programa de formación, por lo tanto, este debe ser considerado como un proceso continuo de recolección y tratamiento de datos, a través de la revisión y seguimiento de las funciones de los profesionales, los avances tecnológicos y las exigencias del sector. Este monitoreo permitirá descubrir variaciones en el proceso de producción de textos y establecer criterios para su avance y reorientación técnica. En cuanto a las empresas, Castaño, López y Prieto (2011) explican cómo estas elaboran un perfil de exigencias para cada cargo o profesional:

Dicha descripción debe estar escrita; siguiendo un formato objetivo para recoger requisitos, contenido y alcance del puesto: qué hace, cómo se hace, por qué, dónde y cuándo se hace: misión/objetivo, funciones y actividades, requisitos (conocimiento, habilidades, experiencias), responsabilidades, relaciones 
jerárquicas y funcionales, condiciones de trabajo. Debe estar actualizada y vigente. Se recomienda su revisión al menos cada dos años (p. 21).

De acuerdo con Gagliardi (2008), los programas de formación deberán diseñar competencias profesionales amplias, que trasciendan las básicas o específicas de la carrera, lo cual propiciará la actualización de las capacidades con el sector empleador en un periodo prudencial. De esta manera, se dispondrá de un porcentaje representativo de aquellas capacidades acordes con la factibilidad de insertar al estudiante en un primer empleo, con lo que este superará la transición de la carrera al mercado de trabajo, es decir, el gran logro se centrará en orientar y disminuir las dificultades laborales y personales que se le puedan presentar al egresado.

En cuanto a las investigaciones relacionadas con la gestión institucional y su vinculación con el contexto, Foncubierta, Perea y González (2016) estiman que, para evaluar la adquisición, el nivel de logro y el desempeño de empleabilidad, resultan imprescindibles, por un lado, las acciones desempeñadas por cada institución educativa de ofrecer una formación específica dirigida a la salida laboral, e impulsar y orientar a cada egresado en este ámbito (formación, emprendimiento y búsqueda del primer empleo, etc.). Por otro lado, consideran necesario potenciar los estudios e informes de empleabilidad que ayuden en la toma de decisiones a fortalecer los puntos fuertes de cada centro educativo, así como solventar los débiles y, de forma especial, estar atentos a las demandas de la sociedad en una espiral continua de evolución, de acuerdo con estudios como el Barómetro de Empleabilidad y Empleo de Profesionales.

De ahí que contar con un programa de atención al primer empleo del egresado, por parte del agente empresarial y el educativo, pueda disminuir la tensión que produce buscar trabajo, lo que irá acompañado de información relevante en pos de arribar a una oportunidad real de incorporación laboral de manera independiente y segura. Este es el momento de otorgar valor al aprendizaje de las competencias genéricas que impulsen al egresado no solo a conseguir empleo, sino a mantenerlo al haber consolidado habilidades de comunicación, de presentación de su marca personal, de afrontar una entrevista, o bien, el desarrollo de un proyecto empresarial propio, como alternativa posible.

Respecto del proceso de reclutamiento y selección del profesional redactor de escritos, puede darse bajo un programa conjunto entre las empresas interesadas en el talento humano y las instituciones terciarias que lo formen; en este empeño compartido, se utilizarán las herramientas tecnológicas más actualizadas con el fin de retroalimentar la información que mejore la calidad de gestión de la producción de textos e impulse la formación de nuevas competencias.

Una instancia esencial en la formación de las competencias de los profesionales de la escritura son las PP, definidas por el ISLEM (2016), como un proceso de construcción de saberes teórico-prácticos y técnicos, que desarrollan los estudiantes, producto de la formación adquirida en los espacios del plan curricular de la tecnicatura y de la composición de situaciones reales, de las relaciones con sus pares y con el futuro ámbito de trabajo. Entre las investigaciones relacionadas con las PP y las exigencias laborales se encuentra la de Visiers (2016), quien destaca que en un sistema de enseñanza integrado debe existir la mayor concordancia posible entre los objetivos formativos, los métodos de enseñanza-aprendizaje y los procedimientos de evaluación. A la vez, los estudios terciarios, consustanciados con esta coherencia, deben privilegiar la formación basada en competencias y en el protagonismo del estudiante, como motor de su aprendizaje; desde este ángulo, las PP pasan a ser el escenario privilegiado para su desarrollo y evaluación, porque se basan en una acción dinámica, 
dialogante, en continuo aprendizaje, donde se entrelazan los espacios curriculares con las experiencias significativas de los cursantes y la realidad del campo profesional.

En cuanto al dominio de competencias, dentro del ámbito técnico profesional, el redactor de textos requerirá desarrollar capacidades eficaces y adecuadas ante situaciones de difícil resolución. Para Tobón, Pimienta y García Fraile (2010), una competencia es entendida como tal cuando existe un desempeño integral para interpretar, argumentar y resolver problemas de contexto con sentido creativo e idoneidad, y a la vez se atienda, de manera articulada, al desarrollo del saber ser, convivir, conocer y hacer para confluir en las parejas idoneidad-mejoramiento, continuo-ética. Por tanto, las competencias se comportan como una actuación integral ante las situaciones del contexto donde se ponen en juego no solo los saberes mencionados, sino también aquellas acciones que otorgan identidad a la socioformación. En la misma línea se encuentra la conceptualización de Tejeda, Sánchez y Mariño (2012), para quienes la competencia es una cualidad humana conformada de manera integral, que fusiona los distintos saberes (conocer, ser y hacer), construidos a través del desempeño eficaz de los recursos personológicos de los profesionales en función de desarrollar su carrera en un ambiente laboral cambiante, dinámico y complejo.

Villegas, Borjas e Icaza (2015) refuerzan la importancia que tiene la implementación del aprendizaje basado en competencias, tanto para el estudiante, al permitirle poner en práctica los conocimientos adquiridos e integrar el saber con el saber hacer, como para el docente, cuyo papel se agiganta en su misión de guiar y formar profesionales competentes conforme a las exigencias del mercado laboral. Coincide con estos resultados el trabajo de González Lorente y Rebollo-Quintela (2018), para quienes la formación inicial de los docentes es esencial en su misión de facilitadores del dominio de competencias, inserción laboral, mejora y actualización permanente de los estudiantes.

Sobre la base de los eficaces resultados del modelo de formación por competencias, la Tecnicatura Superior en Redacción de Textos, impartida por el ISLEM, viene gestando un proceso de cambio para lograr su implementación. En efecto, se ha trabajado en su plan curricular a partir de la toma de conciencia de la cultura organizacional existente con la idea de intervenir en las debilidades y fortalezas, tanto de su cuerpo directivo, como del resto de los miembros de la comunidad educativa, cuyo objetivo se centra en poder abordar, con mayor certeza, el programa de formación. Sin duda, se requiere información, orientación y mejores prácticas centradas en el alumno, además del tiempo, los recursos y las capacidades de los docentes, agentes funcionales, y de los directivos, líderes vigilantes y comprometidos en generar transformaciones reales y monitoreadas con las necesidades del contexto social y laboral. Al respecto, enfatiza Garbanzo-Vargas (2016):

[...] los trabajadores de una organización actúan en distintas modalidades de manera individual y colectiva según su naturaleza. Estas no funcionan al azar y el éxito que se alcanza es producto de un conjunto de sinergias que, conducidas en cierta dirección, lo logran. El éxito no viene en forma aleatoria, las organizaciones son células sociales que responden a estímulos del ambiente tanto interno como externo y, según estos, son sus productos, sus comportamientos (p. 69).

El desarrollo del programa curricular o carrera bajo el modelo de aprendizaje por competencias, al que adhiere el ISLEM, expresado en la Resolución n. ${ }^{\circ}$ 3770/MEGC (2017), requiere de la comprensión y dominio del cuerpo docente, quien adaptará el circuito enseñanza-aprendizaje orientado hacia esa perspectiva. Desde este ángulo, la práctica educativa, ejercida por todos los miembros de la institución, abre la brecha a fin de entender y dominar una nueva manera de producir conocimiento, de inaugurar espacios de participación 
que generen respuestas a las necesidades del mercado laboral y, a la vez, contribuir con la calidad educativa del instituto.

El perfil profesional que plantea la tecnicatura organizada por el ISLEM para la formación de los técnicos redactores está enraizado en la necesidad comunicativa de producir mensajes escritos de circulación social; para tal fin, no solo dominará amplios conocimientos de la lengua en sus distintos planos, sino, además, las técnicas y estrategias de los diferentes tipos de escritos y estilos textuales, amén de las nuevas tecnologías orientadas a la producción y edición de textos.

El programa de formación impartido en la tecnicatura busca concertar el binomio educación formal-trabajo, por ello establece como objetivo ordenar de manera lógica un conjunto de unidades curriculares relacionadas con el campo de la formación en el área de la PP del redactor de textos (PP I, II y III, y PP Integrada). De acuerdo con la resolución citada, la meta es "integrar al estudiante en un contexto de aprendizaje situado en ámbitos reales relacionados con la práctica profesional del redactor de textos" (p. 17), para lo cual se aplican diferentes metodologías como las propias del trabajo de campo, estudios de casos y resolución de problemas en contextos reales.

\section{Método}

Este estudio se propone analizar la percepción de dos variables: a) la que tienen los redactores de textos, egresados del ISLEM, en relación con la pertinencia de las competencias logradas en las PP, y b) la que experimentaron ante las exigencias de la inserción laboral.

\section{Diseño}

Para encarar la correlación de las variables, se acudió a la investigación cuantitativa y se instrumentó el estudio de campo, con el fin de realizar una medición de las variables; el mecanismo no experimental y transversal, y a la vez correlacional, permitió analizar hasta qué punto las variables observadas se correlacionaron, lo que contribuyó a verificar la hipótesis planteada.

\section{Hipótesis}

En este sentido, la hipótesis de investigación llevó a confirmar que la percepción que poseen los redactores de textos sobre el dominio de competencias alcanzado en el momento de las PP está relacionada con el nivel de requerimientos de los empleadores.

\section{Población}

La población de esta investigación estuvo representada por 51 (cincuenta y un) técnicos superiores, egresados de la Tecnicatura Superior en Redacción de Textos, cursada en el ISLEM desde la primera cohorte de exalumnos de 2008 hasta julio de 2019. En este estudio se tomaron a todos individuos que egresaron de la tecnicatura, quienes representan el universo sobre el que se trabajó.

\section{Instrumento}

El cuestionario se estructuró en 26 (veintiséis) preguntas, en una escala de actitudes de cinco opciones (la opción 1 representa la escala más baja y la 5, la más alta); los egresados señalaron, en cada una de las competencias, el nivel de desarrollo que alcanzaron cuando culminaron las PP y el nivel de esas competencias como exigencia para insertarse en los puestos de trabajo como redactores de textos. 
El proceso de validación del instrumento, encaminado a confirmar la validez del contenido y la estructura, se realizó a través de la técnica del Juicio de Experto. Para tal fin, se solicitó la revisión de profesores investigadores de la Universidad Internacional Iberoamericana (UAI), de la Argentina, pertenecientes al Departamento de Ciencias del Lenguaje, Educación y Comunicaciones, del Doctorado en Educación. De este modo, los expertos evaluaron, a partir de sus perspectivas, como pertinentes las dimensiones e indicadores seleccionados, así como la forma en que están medidos. Para realizar dicha validación, se les remitió, a través de la cuenta del correo electrónico, no solo el cuestionario construido, sino, además, un resumen del problema, los objetivos generales y específicos a fin de que pudieran contextualizar el instrumento elaborado. Una vez devuelto, se analizaron los alcances de la evaluación con el objetivo de incorporar las recomendaciones emitidas; entre ellas: reformulación de ítems para esclarecer su contenido; supresión de algunos por su semejanza con otros o por su inutilidad respecto de la recolección de datos. La investigadora tuvo en cuenta las observaciones para la mejora del cuestionario.

En una segunda instancia, se procedió a determinar su consistencia interna a través de la aplicación de un cuestionario inicial al grupo piloto, del cálculo del coeficiente de confiabilidad del alfa de Cronbach con la fórmula correspondiente. Para esta se empleó la Hoja de Cálculo de Excel a fin de computar estimaciones de confiabilidad de instrumentos, aprobada y aceptada por Neag School of Education, el cual arrojó como resultado 0,82. Para contestar la pregunta de investigación, se utilizaron estadísticos descriptivos de frecuencia, el por ciento y medias, y, para las hipótesis, la estadística correlacional bivariadas sobre la base del Programa Statistical Program for Social Sciences (SPSS), versión 19.0.

\section{Procedimiento de la investigación}

En la primera fase, en correspondencia con el diseño de la idea de investigación, se realizó un diagnóstico informal previo, el que consistió en delimitar el objeto de estudio, logrado a través de entrevistas informales a diferentes actores de la comunidad educativa del ISLEM, con preferencia a los estudiantes y egresados, para conocer sus inquietudes con respecto a las competencias adquiridas durante las PP y durante toda la carrera; además, se realizó el arqueo, clasificación, organización y análisis de diversos documentos institucionales, informes de trabajos científicos precedentes en revistas especializadas del área de las ciencias sociales, educativa y gerencial. De acuerdo con la revisión de la literatura, se decidió configurar las variables: competencias y las exigencias laborales, cada una con sus respectivas dimensiones e indicadores, ya descritas en la explicación de los pasos para la construcción del instrumento.

En la segunda fase, se diseñó una técnica e instrumento para medir la percepción que tenían los egresados en cuanto a la relación entre la adquisición de competencias durante la formación de la carrera y las exigidas en el ámbito laboral. Para tal fin fue necesaria la construcción del instrumento, a partir del diseño del mapa de variable, la elaboración del cuestionario, la validación mediante la técnica juicio de experto y la confiabilidad del instrumento.

Durante la tercera fase, se determinó la población, la cual se identificó como finita y pequeña, por lo tanto, no se requirió la aplicación de ninguna técnica de muestreo, aunque fue necesario ubicar, en lo posible, a todos los egresados de la carrera de redacción de textos.

En la cuarta fase, se llevó a cabo la aplicación del instrumento, el cual se administró en las modalidades presencial, 38 (treinta y ocho) integrantes, y a distancia, 13 (trece), a través del correo electrónico; de esta manera, se concretó el llenado de cuestionarios. 
En la quinta fase, se analizaron los resultados de los instrumentos, a través del tratamiento estadístico por medio del programa SPSS, versión 19.0., donde se observaron las relaciones entre las variables, lo cual permitió realizar la verificación de la hipótesis de investigación establecida.

En la sexta fase, se formuló la propuesta, cuyo objetivo general aconsejó el diseño de un programa de formación continua por competencias, relacionadas con el trabajo en red, emprendimiento, marca personal y dominio de tecnologías ajustadas a los cambios que se dan en el mercado laboral e indispensable para la inserción laboral del egresado de la tecnicatura en estudio.

\section{Resultados}

Los resultados se obtuvieron en función de los puntajes de cada una de las competencias que el redactor de textos señaló a partir de la percepción de ambas variables: a) el dominio en las PP y b) la exigencia laboral. Por lo general, los resultados, en todas las competencias medidas, fueron mayores en la percepción de la exigencia laboral que las logradas en las PP, como se observa en la tabla 1.

Tabla 1

Frecuencias absolutas y relativas de ambas percepciones

\begin{tabular}{|c|c|c|c|c|c|c|}
\hline Competencias & Variable & $\begin{array}{c}\text { Valor } \\
1 \\
\end{array}$ & $\begin{array}{c}\text { Valor } \\
2 \\
\end{array}$ & $\begin{array}{c}\text { Valor } \\
\mathbf{3} \\
\end{array}$ & $\begin{array}{c}\text { Valor } \\
4 \\
\end{array}$ & $\begin{array}{c}\text { Valor } \\
5 \\
\end{array}$ \\
\hline \multirow{2}{*}{ Gramaticales } & PP & $0 \%$ & $0 \%$ & $3,9 \%$ & $14,2 \%$ & $81,5 \%$ \\
\hline & Inserción laboral & $0 \%$ & $0 \%$ & $0 \%$ & $10,4 \%$ & $89,6 \%$ \\
\hline \multirow[t]{2}{*}{ Textuales } & $\mathrm{PP}$ & $0 \%$ & $0 \%$ & $5,8 \%$ & $36,4 \%$ & $57,8 \%$ \\
\hline & Inserción laboral & $0 \%$ & $0 \%$ & $0,1 \%$ & $29,6 \%$ & $70,3 \%$ \\
\hline \multirow{2}{*}{$\begin{array}{l}\text { Enciclopédicas } \\
\text { e información }\end{array}$} & PP & $0 \%$ & $2,7 \%$ & $28,2 \%$ & $32,8 \%$ & $36,8 \%$ \\
\hline & Inserción laboral & $0 \%$ & $0 \%$ & $0,8 \%$ & $18,4 \%$ & $80,8 \%$ \\
\hline \multirow[t]{2}{*}{ Generales } & PP & $0 \%$ & $1 \%$ & $19,1 \%$ & $44,6 \%$ & $35,3 \%$ \\
\hline & Inserción laboral & $0 \%$ & $0 \%$ & $2,9 \%$ & $33,4 \%$ & $63,7 \%$ \\
\hline
\end{tabular}

Nota. Elaboración propia, basada en el tratamiento estadístico de datos. El egresado valoró cada percepción de 1 a 5; Valor 1: bajo dominio de competencia en las PP o exigencia laboral; Valor 5: alto dominio de competencia en ambas variables.

Luego se realizaron los cálculos para saber si existían diferencias entre las percepciones del dominio de competencias en ambas valencias. Al efectuar las sumatorias de valoraciones para cada una de las competencias del redactor de textos, se comprobó que ambas tenían comportamiento normal; no obstante, no se evidenciaron diferencias estadísticas significativas entre los puntajes de las dos percepciones en cada una de las competencias medidas, como se muestra en la tabla 2. 
Tabla 2

Sumatorias medias de ambas percepciones

\begin{tabular}{lcc}
\hline Competencias & $\begin{array}{c}\text { Prácticas } \\
\text { profesionalizantes }\end{array}$ & Inserción laboral \\
\hline Gramaticales & 4,77 & 4,89 \\
Textuales & 4,47 & 4,90 \\
Enciclopédicas e información & 4,30 & 4,80 \\
Generales & 3,90 & 4,50 \\
Percepción Total & 4,20 & 4,70 \\
\hline
\end{tabular}

Nota: Elaboración propia, a partir de los datos obtenidos del tratamiento estadístico.

Como se observa en los resultados de la tabla 2, las puntuaciones medias alcanzadas en la medición de percepciones de dominio de las competencias para la inserción laboral superan los valores logrados en la medición de percepciones del dominio de competencias al culminar las PP.

Sin embargo, se evidencia, que las competencias generales son las que presentan mayores diferencias en las medias obtenidas en el instrumento aplicado a los egresados sobre ambas percepciones. Si estas se analizan, se advierte su afinidad al no presentar diferencias significativas. En la tabla 3 se presentan los resultados de la aplicación del estadístico " $\mathrm{t}$ " de Student.

Tabla 3

Relación entre percepción del dominio de competencias del redactor de textos logradas en las PP y las exigidas en la inserción laboral

\section{Competencias}

Gramaticales

Textuales

Enciclopédicas e información

Generales

\section{Coeficiente de correlación}

0,673

0,713

0,404

0,233
Significancia bilateral

0,010

0,001

0,119

0,171

Nota: Elaboración propia, a partir de los datos obtenidos del tratamiento estadístico.

En síntesis, los valores alcanzados demuestran que las relaciones entre las competencias específicas del redactor de textos (gramatical, textual, enciclopédica e información general), adquiridas en el centro educativo, son levemente mayores a las exigidas, del mismo tenor, en el ejercicio laboral. A su vez, todas son significativas al nivel de 0,05 o inferior, de carácter positivo. Por lo tanto, el análisis permite indicar que existe una relación entre ambas percepciones. 


\section{Discusión y conclusiones}

La hipótesis de investigación se orientó a averiguar si la percepción que poseen los redactores de textos acerca del dominio de competencias alcanzado durante su formación está relacionada con la percepción de dominio de conocimientos y habilidades requeridas en la inserción laboral. A partir de los datos obtenidos en cada una de las competencias consultadas, se pudo demostrar que existe relación entre ambas percepciones; en ese sentido, todas las competencias adquiridas durante la formación recibida durante el proceso de las PP son requeridas en el mercado laboral para el ejercicio de la redacción, pero con mayores valores de exigencias.

En consecuencia, los resultados demuestran coherencia entre el plan de estudio de la Tecnicatura Superior en la Redacción de Textos, del Instituto Superior de Letras Eduardo Mallea, y el sector laboral donde ejercen los egresados de la institución, entre las que se encuentran, en forma mayoritaria, empresas del sector comunicación de la Ciudad Capital y la Provincia de Buenos Aires, Argentina.

Al tratar de manera individual cada una de las competencias consultadas en el instrumento y buscar las relaciones entre ambas percepciones, se verificó que el grupo de competencias con mayores diferencias en cuanto a los valores de exigencias del sector laboral son las competencias generales relacionadas con integración a equipos profesionales, trabajo colaborativo, creatividad y proactividad.

Paralelamente, las competencias con menores diferencias en los valores de exigencias del sector laboral y las logradas durante la capacitación profesional son las gramaticales, textuales, enciclopédicas e información, que forman parte del eje central de la tecnicatura y están vinculadas de manera directa con las funciones profesionales.

Estas diferencias pueden estar asociadas a la falta de experiencia, es decir, que las PP no garantizan la maestría suficiente para que los empleadores decidan a favor de la contratación de un profesional recién egresado. Marrero (2004) plantea que la falta de experiencia previa en las carreras es uno de los aspectos que vuelve más dificultoso el ingreso de los jóvenes en el mercado del trabajo y cuestiona la imposibilidad de poder cumplir con ese requisito al ser exigido siempre. A pesar del tiempo transcurrido -la autora lo plantea en 2004- la experiencia sigue siendo una limitante para el nuevo profesional que, si bien cuenta con un título oficial, no resulta suficiente a la hora de llenar una vacante en un empleo para el que fue calificado.

En cuanto a la implicación de los datos obtenidos en la presente investigación, es importante destacar que, para el ISLEM puede resultar de gran utilidad aplicar los resultados para una revisión del plan de estudio de la carrera de redactor de textos, donde primarán los ajustes necesarios a las actividades y estrategias empleadas por los profesores durante las PP, con el fin de perfeccionar el dominio de cada una de las competencias que están relacionadas directamente con la profesión.

Sin duda, será de sumo interés para el instituto considerar también la viabilidad de redimensionar las competencias generales en el perfil del redactor de textos, en pos de ofrecer una mayor apertura en su accionar profesional. En forma paralela considerará armonizar con la oferta de un programa de formación continua, acorde a las actuales exigencias laborales donde se posibilite, no solo contar con una formación profesional formal, vinculada con las competencias gramaticales, textuales, enciclopédicas y de información, sino también con una formación permanente que lo ayude a consolidar su marca personal, buena reputación y alta calificación para la inserción en lugares clave que soliciten su participación. Todo esto sin 
perder de vista que los desafíos laborales de los tiempos que corren son sumamente exigentes para acceder a trabajos cualificados.

Sobre la base de los planteamientos del párrafo anterior, se diseñó una propuesta, la cual tiene como objetivo general el diseño de un programa de formación continua de competencias relacionadas con el trabajo en red, el emprendimiento, la marca personal y el dominio de tecnología, ajustados a los cambios que se dan en el trabajo e indispensables para la inserción laboral del egresado.

De acuerdo con los resultados encontrados en la investigación, es positivo tener identificadas las competencias que presentan deficiencias en el programa de formación del profesional de redacción textual, a partir de las exigencias actuales en el ámbito laboral; sin embargo, la mayor limitación para la mejora del plan de estudio es la burocracia en los procesos administrativos ante los entes gubernamentales que aprueban los diseños curriculares.

No obstante, y a pesar de tener focalizado el problema, surgen nuevos interrogantes: ¿están las organizaciones de educación técnica profesional en capacidad de propiciar los cambios de gestión administrativa y académica que permitan el enfoque por competencias? También se hace necesario la revisión de ciertas variables relacionadas con la formación continua de los docentes, la estructura de trabajo en equipos para la elaboración de proyectos, la reacción de los docentes sobre nuevas metodologías, la revisión permanente de las competencias y el trabajo en alianza con los entes involucrados en la actualización del perfil profesional.

\section{Referencias}

Castaño, M., López, G. y Prieto, J. (2011). Guía técnica y de buenas prácticas en reclutamiento y selección de personal. Publicación Digital del Colegio de Oficial de Psicólogos de Madrid. http://www.copmadrid.org/webcopm/recursos/guiatecnicabuenaspracticas.pdf

Di Meglio, G.; Barge-Gil, A.; Camiña, E. y Moreno, L. (2019). El impacto de las prácticas en empresas en la inserción laboral: un análisis aplicado a los grados de Economía y Administración y Dirección de Empresas. Educación XX1, 22(2), 235-266. https://doi.org/10.5944/educXX1.22579

Fascendini, P.; Guaita, M.; Peralta, J. y De Gennaro M. (2019). El impacto de los espacios formativos acerca de las competencias sociales y laborales en la carrera de medicina veterinaria. Revista FAVE -Sección Ciencias Veterinarias, 18 (2019) 40-44; https://doi.org/10.14409/favecv.v18i2.8505

Florez, M.; Luraschi, M. y Butti, F. (2017). Las Prácticas Profesionalizantes en la formación de jóvenes que asisten a Escuelas Técnicas en dos localidades de la provincia del Chaco. El caso de la orientación "Madera y Mueble" y "Construcciones". In Congreso Nacional de Estudios del Trabajo. Buenos Aires. https://www.aset.org.ar/2017/ponencias/12_Florez.pdf

Foncubierta, M.; Perea, J. y González, G. (2016). Una Experiencia la Universidad en la vinculación universidad empresa: El Proyecto COGEMPLEO de la Fundación Campus Tecnológico de Algeciras. Educación XX1, 19(1), 201-225. 
Gagliardi, R. (2008). Gestión de la educación técnica profesional. Formación de competencias profesionales. Capacitación directiva para la formación de jóvenes autónomos. Noceduc.

Garbanzo-Vargas, G. (2016). Desarrollo organizacional y los procesos de cambio en las instituciones educativas, un reto de la gestión de la educación. Revista Educación, 401, 67-87. https://www.redalyc.org/pdf/440/44043204005.pdf

González, J. (2016) El Análisis Situacional del Trabajo, una alternativa para el desarrollo de los planes de estudios de la Ingeniería en Energías Renovables en la Universidad Tecnológica de Altamira. RIDE, Revista Iberoamericana para la Investigación y el Desarrollo Educativo, 6(12). http://www.redalyc.org/articulo.oa?id=498153966014

González Lorente, C. y Rebollo-Quintela, N. (2018). Competencias para la empleabilidad de los futuros maestros de Educación Primaria: Una mirada a su proceso de inserción socio-laboral. Revista Digital de Investigación en Docencia Universitaria, 12(2), 114131. http://dx.doi.org/10.19083/ridu.2018.730

Instituto Nacional de Educación Tecnológica (INET) (2019). Prácticas profesionalizantes. https://bit.ly/3ahv4Fa

Instituto Superior de Letras Eduardo Mallea (ISLEM) (2016) Prácticas Profesionalizantes del Redactor I. Resolución de problemas. Enfoques teóricos y orientaciones metodológicas para las Prácticas Profesionalizantes del Redactor. Ediciones Mallea.

Marrero, A. (2004). Buenos, bonitos y baratos. Las expectativas empresariales sobre los jóvenes y la educación en Uruguay. Universidad de la República O. del Uruguay. ${ }^{\text {to. }}$ de Sociología y Economía de la Educación (FHCE). Cuadernos de Relaciones Laborales, 22(2), 137-165. https://dialnet.unirioja.es/servlet/articulo?codigo=1096916

Pedreño, A. (2017). La digitalización y la economía global. Visión general. En: El Cambio Digital en la Economía. Un proceso disruptivo. Revista ICE Información Comercial Española, $\quad$ 897, 9-22. http://www.presidencia.gva.es/documents/166658342/166725599/Ejemplar+897/a5705 a 87-5cd7-400e-b055-34e103eb10ab

Resolución n. ${ }^{\text {o }}$ 3770-MEGC (2017) Plan de Estudio de la Tecnicatura Superior en la Redacción de Textos, del Instituto Superior de Letras Eduardo Mallea (ISLEM). Gobierno de la Ciudad Autónoma de Buenos Aires. Ministerio de Educación de la República Argentina (Opción pedagógica Distancia).

Silva et al. (2019). Perfil de los graduados en enfermería: competencias e inserción profesional. Revista Latino-Americana de Enfermagem, 27, e3205. https://doi.org/10.1590/1518-8345.3222.3205

Tejeda Díaz, R.; Sánchez, P. y Mariño, M. (2012). Gestión formativa competente de pro en la educación superior. Universidad Holguín, Cuba. Centro de Estudios sobre ciencias de la educación. Red Durango de Investigadores Educativos Experiencias de aplicación de las competencias en la educación y el mundo organizacional (pp. 201-243).

Tobón, S.; Pimienta, J. y García Fraile, J. A. (2010). Secuencias didácticas: aprendizaje y evaluación de las competencias. Editorial Prentice Hall.

Tomassiello, R. y Zani, V. (2016). Formación Profesional e inserción laboral en artes, diseño y humanidades. Estrategia para una articulación efectiva. Huellas, Búsquedas en Artes y Diseño, 9, 52-61. https://bdigital.uncu.edu.ar/objetos digitales/8667/06-tomassiellohuellas9-2016.pdf 
Villegas, F.; Borja, E. e Icaza, D. (2015). Competencias y habilidades desarrolladas en los estudiantes de Contabilidad y Auditoría: Diagnóstico de la Universidad Estatal del Milagro. Vcuador. Visión Empresarial, 5, 113-134. https://doi.org/10.32645/13906852.418

Visiers, L. (2016). Evaluación de competencias en las prácticas tuteladas del Grado en Enfermería. (Tesis doctoral) Universidad Europea de Madrid (España). https://dialnet.unirioja.es/servlet/tesis? codigo $=81802$

Fecha de recepción: 02/09/2020

Fecha de revisión: 20/04/2021

Fecha de aceptación: 13/07/2021 